\title{
Peak-Fitting of High Resolution ToF-SIMS Spectra: A Preliminary Study
}

\author{
M-L. Abel*, K. Shimizu, M. Holliman and J.F. Watts \\ The Surface Analysis Laboratory, Faculty of Engineering and Physical Sciences, \\ University of Surrey, GU2 7XH UK
}

\begin{abstract}
Peak-fitting has been performed on a series of peaks obtained by ToF-SIMS analysis in order to assess whether information may be obtained from this procedure on the samples' characteristics. A variety of samples were examined including a range of treatments for aluminium leading to different surface roughnesses, polymer films with a range of polydispersities, molecular weight and thicknesses as well as aluminium samples with adsorbed adhesion promoters on the surface. Variation of peak-fitting was assessed by varying the peak intensity, full width at half maximum and peak asymmetry.

Although further studies are needed it is possible to say that the peak width increases with roughness whereas peak asymmetry seems to be related to oxide thickness. Polymer characteristics do not seem to influence the width whereas the peak asymmetry increases either versus molecular weight or polydispersity. A possible assumption is that the peak asymmetry relates to the ion formation processes. Additional work with varying polymer films thickness indicates that both FWHM and peak asymmetry may be related to sample charging and this could be used for assessment of film thicknesses. Finally, peak-fitting was used to obtain a more reliable peak area when peaks are too close in mass to use current methods.
\end{abstract}

Keywords: Peak-fitting, ToF-SIMS, roughness, charging, quantification

*email: m.abel@surrey.ac.uk 


\section{Introduction}

Peak-fitting is of the utmost importance when examining spectroscopic signals that may not be entirely resolved. This is widely used for X-ray photoelectron spectroscopy (XPS), for instance, in order to identify which chemical species are present within a convoluted spectrum. The parameters that influence the shape and width of the peaks are well known and include pass energy, roughness of the sample, $\mathrm{X}$-ray line width and so on. Such a procedure is not usually employed in time of flight secondary ion mass spectrometry although this technique would generally benefit from it, indeed, current modern equipment is capable of generating spectra with small peak widths and very high resolution. Data such as the width and asymmetry of the peaks could be used to correlate the aspect of high resolution spectra with sample characteristics or even fragmentation phenomena. These include sample topography, peak rearrangement in the selvedge region or surface chemistry of the sample.

In the work presented here, two examples are given whereby variation of either peak width or asymmetry or both may be observed as a function of a selection of parameters including sample roughness and molecular weight of a polymer. A third example is given showing that peak intensity may be obtained using peak-fitting when several peaks are very close in mass and is subsequently used for adsorption studies.

\section{Experimental}

Samples of aluminium in a range of roughnesses, from diamond polished aluminium to grit-blasted aluminium were examined (degreased aluminium (Goodfellows, Cambridge Ltd.), grit-blasted aluminium (with $50 \mu \mathrm{m}$ alumina grit), heated aluminium $\left(220^{\circ} \mathrm{C}, 2\right.$ hours $)$, diamond polished aluminium, shiny side and matt sides of aluminium foil). Polystyrene (PS) (Pressure Chemical Company) samples were dissolved in acetone until solution saturation. PS samples were deposited on aluminium substrates by spin coating. $1.7 \times 10^{-5} \mathrm{M}, 8.7 \times 10^{-5} \mathrm{M}$ and $3.4 \times 10^{-4} \mathrm{M}$ concentrations, with molecular weight (MW) ranging from 90,000 to $950,000 \mathrm{~g} \mathrm{~mol}^{-1}$ were used to produce different thicknesses of the PS layer. The polydispersity of the three different types of PS ranged from 1.04 to 1.10 .

Aluminium sheets (Goodfellow Cambridge Ltd.) were washed using a Scotchbrite pad with detergent and tap water, in order to partially remove the oxide layer, followed by rinsing with acetone. The aluminium sheet was then grit-blasted with 50 $\mu \mathrm{m}$ alumina grit. Discs of $10 \mathrm{~mm}$ in diameter were punched from aluminium sheets and subsequently cleaned with acetone, using an ultrasonic bath, to remove any contamination. Solutions of $\gamma$-glycidoxy propyl trimethoxy silane (GPS, A187 GE Specialty Materials) were prepared in deionised water in the range $4.5 \times 10^{-1}$ to $4.5 \mathrm{x}$ $10^{-8} \mathrm{M}$. The solution was then left to hydrolyse for an hour with stirring. Grit-blasted aluminium samples were dipped in $20 \mathrm{~cm}^{3}$ of the candidate GPS solution for 10 minutes. The samples were then immediately rinsed with deionised water twice for 5 minutes, using an ultrasonic bath. Duplicate samples were prepared for XPS and ToFSIMS analysis at each concentration.

Surface analysis was performed on a TOF-SIMS 5 (ION TOF GmbH, Münster Germany). Static SIMS with an ion dose of less than $1 \times 10^{13}{\text { ions } \mathrm{cm}^{-2} \text { analysis }}^{-1}$ was employed using either a $\mathrm{Bi}^{+}$or a $\mathrm{Bi}_{3}{ }^{+}$primary ion beam operating in the high current bunched mode, at a pulse width of $25 \mathrm{~ns}$ and a pulse frequency of $10 \mathrm{kHz}$. The 
analysis area was $100 \times 100 \mu \mathrm{m}^{2}$ and the resolution $64 \times 64$ pixels. SIMS spectra were acquired over a mass range of 0-850 $\mathrm{u}$ in both positive and negative ion modes.

Peak-fitting of the high resolution spectra was carried out using CASAXPS software v.2.3.14 (CASA Software Limited, Torquay Devon). All data presented are the results of two different analyses on the same sample. The line shape used was a combination of $70 \%$ Lorentzian and $30 \%$ Gaussian established by trial and error on a symmetrical spectrum. The asymmetry index was given by:

$$
a=1-\frac{H W H M_{r i g h t}}{H W H M_{\text {left }}} \quad<1>
$$

Where $\mathrm{HWHM}_{\text {right }}$ and $\mathrm{HWHM}_{\text {left }}$ are the half width at half maximum on the right and on the left of asymmetric peaks respectively. Goodness of fit was established by eye, for the reasons outlined by Leadley and Watts for electron spectra [i, ii].

\section{Results}

\section{Case-study 1: roughness of aluminium}

Samples were examined with both $\mathrm{Bi}^{+}$and $\mathrm{Bi}_{3}{ }^{+}$primary ion beams, in addition peakfitting was performed for three ions: $\mathrm{H}^{+}, \mathrm{Al}^{+}$and $\mathrm{Al}(\mathrm{OH})_{2}{ }^{+}$. As all peaks examined here were fitted with the same shape, only two parameters are available for comparison: peak width described as the usual full width at half maximum (FWHM) and the peak asymmetry. Table 1 shows the FWHM and asymmetry parameter $a$ for $\mathrm{Al}^{+}$and $\mathrm{Al}(\mathrm{OH})_{2}{ }^{+}$for samples analysed with $\mathrm{Bi}_{3}{ }^{+}$. The biggest width is seen for gritblasted aluminium. The effect of roughness is well known in surface analysis in that it usually degrades resolution, in this case mass resolution, and may result in an increase in width of peaks. It is known that a grit-blasted aluminium will exhibit a roughness of a few microns [iii] and is most likely the roughest surface being examined here. A large width is also observed for the heated aluminium and it is known that a heat treatment may increase roughness too. However, another likely factor to contribute to a large increase of FWHM is probably the thickness of the oxide. Analyses have been performed with a unique flood gun setting $(20 \mathrm{eV})$ and may probably be improved with a variable energy addition to the instrument (now available from ION TOF).

If we now examine the data obtained for the asymmetry parameter of the fitting, $a$, similar results to the width were obtained, with a marked difference for the heated sample which exhibits the largest asymmetry of all samples for both $\mathrm{Al}^{+}$and $\mathrm{Al}(\mathrm{OH})_{2}{ }^{+}$. For both types of primary ion beams the smallest asymmetry was obtained for either polished or matt side of the aluminium foil for all three ions. This shows that both asymmetry and FWHM may be correlated to the oxide thickness and roughness and hence could be correlated to actual measurable characteristics of materials if plausible models are used. 


\section{Case study 2: effect of polymer polydispersity, molecular weight and film thickness}

Peak-fitting was performed on two types of samples: either a very thick film of PS was spin cast on an aluminium substrate and three peaks were chosen as $\mathrm{H}^{+}, \mathrm{C}_{6} \mathrm{H}_{5}{ }^{+}$ and $\mathrm{C}_{8} \mathrm{H}_{7}{ }^{+}$to compare the effect of molecular weight and/or polydispersity on the peak shapes or three different thicknesses were cast to check the effect of thickness on the signal. The latter experiment will only be described for $\mathrm{Bi}_{3}{ }^{+}$. Originally this polymer was chosen because it is known that when ToF-SIMS analysis is performed on PS some of the ions yielded result from rearrangement in the selvedge region (aromatic polycyclic) and can therefore engender ions with a wider distribution of flight times and hence a larger asymmetry of peaks. However, at this stage only lower mass ions have been fitted and only those results are provided here.

The FWHM did not highlight any particular behaviour, trend or differences amongst samples. When the asymmetry is examined (see Table 2), a few trends can be observed: overall $a$ can be ranked in the same order as the molecular weight or incidentally the polydispersities of the three types of PS used in this work. Incidentally as the spectra all exhibit a signal without aluminium $\mathrm{Al}^{+}$at nominal mass 27 a variation of peak shape cannot be attributed to charging in any way. This indicates that either the molecular weight or the polydispersity have an influence on the asymmetry of the fitted peaks. An obvious explanation may find its origin in the ion formation process which would be slightly different according to these parameters.

Interesting data were obtained when examining the effect of film thickness on fitting parameters. The set of samples was prepared using three different concentrations of PS $(M W=230,000)$ spin cast on an aluminium substrate. Figure 1 shows the signal at mass 27: the $\mathrm{Al}^{+}$signal of aluminium is either intense, well reduced in intensity or absent all together. This confirms a variation of the thicknesses which may be approximately estimated to $2 \mathrm{~nm}, 5 \mathrm{~nm}$ and more than $10 \mathrm{~nm}$. In Figure 2 are shown the values of $a$ for $\mathrm{C}_{6} \mathrm{H}_{5}{ }^{+}$and $\mathrm{C}_{8} \mathrm{H}_{7}{ }^{+}$. In all cases the largest values are obtained for the intermediate thickness films and the same behaviour is obtained for FWHM (not shown here). It is quite likely, in this particular case that the variations may be attributed to a simple charging effect. A thin polymer film deposited on a metal can still benefit from electrons travelling from the substrate, a thick film can benefit from the use of a flood gun whereas for an intermediate thickness film; charge compensation might be slightly more difficult as the film itself will exhibit vertical inhomogeneity in terms of charging which in turn can lead to a slight increase in width and peak asymmetry. This may find further use in assessing a film thickness by calibrating the width obtained for a certain film thickness against both angled resolved $\mathrm{X}$-ray photoelectron data and peak-fitting of ToF-SIMS data.

\section{Case study 3: the use of peak-fitting for adsorption studies}

In this case, peak-fitting has been used in order to obtain intensities of very closely spaced peaks with improved certainty. Indeed, if a silane is deposited on an aluminium substrate it usually forms a covalent bond which in turn produces an $\mathrm{AlOSi}^{+}$cluster ion. This ion is very close in mass to $\mathrm{Al}_{2} \mathrm{OH}^{+}$, the mass difference being only of $12.4 \mathrm{mu}$. It is therefore difficult to obtain its correct intensity using a simple measure of intensity (integrated area beneath an envelope on particular channels). Figure 3 shows a peak-fit of the nominal mass at which the peaks of 
interest appear at nominal mass 71 . Subsequently the intensity may be used and the relative peak intensity (RPI) calculated as follows:

$$
R P I_{x}=\frac{I_{x}}{I_{\text {Total }}} \quad<2>
$$

Where $\mathrm{I}_{\mathrm{x}}$ stands for the integrated intensity of the peak at mass $x$ and $\mathrm{I}_{\text {Total }}$ is the intensity of the total ion signal. This method is a rather crude, univariate type of data transformation and is valid to compare concentrations for adsorption where the matrix effects are negligible. This has been used to compare the intensity of $\mathrm{AlOSi}^{+}$to $\mathrm{Si}^{+}$, i.e. the intensity of covalent bonds to the total amount of silicon containing species on the surface. It was possible to correlate the two intensities up to a certain concentration of GPS silane after which any discrepancy was interpreted as a saturation of covalent formation replaced by an acid-base type of interaction or a change in conformation of the adsorbed molecule [iv]. Besides and because the goodness of fit can also be appreciated "by eye" [i, ii], such peak-fitting may not only bring out an improvement in the quantification of peak intensities but also may render obvious the presence of weak intensity peaks which may otherwise not been observed.

\section{Discussion}

Many factors may influence peak shape including roughness, shape, combination of insulating and conducting materials within the same sample, in other words intrinsic characteristics of the sample that may influence local potential field shapes and hence the trajectories of the ejected ions. Other parameters may include the energy distribution of the ions, as for example elemental ions and cluster ions follow a different energy distribution but also the formation of the ions. It is known that the asymmetry of the peaks may be related to their lifetime and it is therefore anticipated that aromatic polycyclic ions formed from PS will exhibit a high asymmetry. At this stage, it is not known what intrinsic shape any peak should have if it only resulted from the instrumentation present in a TOF-SIMS 5, the only variation for the samples examined here has consisted of using or not the charge compensation facility and setting up the reflector voltage at different values as well as using either $\mathrm{Bi}^{+} \mathrm{Or} \mathrm{Bi}_{3}{ }^{+}$as primary ion beam. All fittings were prepared using only one type of shape as $30 \%$ Gaussian and $70 \%$ Lorentzian, this in itself leaves "room for improvement" but all spectra could be fitted with this one shape. One should note also that a deliberate choice to present data deemed more reliable (reproducible) has been made in order to eliminate gross errors within peak-fitting or possibly erroneous instruments settings. The "goodness" of the fit was checked visually only.

Nevertheless, the observations made above show that a peak-fitting routine incorporated within a data treatment software may be extremely useful. Differences in FWHM and peak asymmetry can be seen which may be interpreted in terms of sample roughness, sample charging and even ion formation phenomena. Such a routine may also improve quantification as well as render obvious the presence of minor peaks. At this stage it is only possible to point out these possibilities, but with sufficient research leading to appropriate models, one might expect to be able to correlate physical material characteristics to their spectra by using a simple but robust technique. These may include oxide thickness, sample roughness, polymer molecular 
weight or polydispersity. More physical information may be obtained too by relating fitting parameters with ion formation process.

Sample roughness, for example, has a well known effect on the flight time of ions because of the different relative starting positions which produce a spread in times. The uppermost surface also creates a screening effect such that some ions travel more slowly than others before they finally reach the extraction optics. Such phenomena have been simulated using the SIMION software and are usually reported in terms of loss of resolution $[\mathrm{v}]$. The work shown here shows that it may be possible to actually quantify the degradation of the spectral resolution by using the FWHM as well as the asymmetry of the peak and ideally relate, maybe even correlate, those data with actual roughness values.

\section{Conclusions}

Peak-fitting have been performed on the ToF-SIMS spectra obtained for various samples in order to assess whether parameters such as sample roughness, polydispersity, to name but a few, could have an influence on peak shape. The following results were obtained:

- Peak width increased with roughness, which is already known but may also be related to oxide thickness whereas peak asymmetry seemed to be mostly related to oxide thickness.

- Polydispersity or molecular weight did not seem to influence the FWHM. However the asymmetry of peaks increased with polydispersity/molecular weight. This may be related to ion formation processes. For different thickness films, the asymmetry and FWHM vary and were the highest for an intermediate thickness film where the charge compensation was probably not as effective.

- Peak-fitting used for adsorption or for more complicated systems may provide more reliable/accurate quantification as well as allowing the observation of minor peaks.

- The use of peak-fitting showed that fitting parameters may be directly correlated with characteristics of the sample itself as well as ion formation phenomena. Appropriate models are required but there is a hope that such parameters may be reliably correlated to sample properties.

\section{Ackowledgements}

MLA acknowledges the Royal Society for provision of a Royal Society University Fellowship. Dr Ian Fletcher is thanked for agreeing to sponsor this work through the "Rapid Communications" channel of Surface and Interface Analysis. Dr Ian Gilmore is acknowledged for useful discussion and advice and Neal Fairley for his help in using CASA XPS software. 


\section{Captions to Figures}

Figure 1: different thicknesses of PS coating illustrated by the variation of the high resolution signal at nominal mass 27 .

Figure 2: asymmetry of peaks $\mathrm{C}_{6} \mathrm{H}_{5}{ }^{+}$and $\mathrm{C}_{8} \mathrm{H}_{7}{ }^{+}$as a function of film thickness.

Figure 3: peak-fitting of nominal mass 71 for an aluminium sample treated with GPS solution $\left(4.5 \times 10^{-1} \mathrm{M}\right)$. The red envelope is the reconstructed signal.

\section{List of Tables}

Table 1: summary of FWHM and peak asymmetry for aluminium samples (signals recorded with $\mathrm{a} \mathrm{Bi}_{3}{ }^{+}$primary ion beam).

Table 2: summary of FWHM and peak asymmetry for polymer samples (solution concentration $3.4 \times 10^{-4} \mathrm{M}$ for all samples).

Amount of word equivalent for Figures and Tables $=5 \times 250=1250$ words Amount of words in main text $=2713$

Total = 3963 (less than 4000 words as indicated in Rapid Communications Rules) 
Figure 1: Different thicknesses of PS coating illustrated by the variation of the high resolution signal at nominal mass 27.

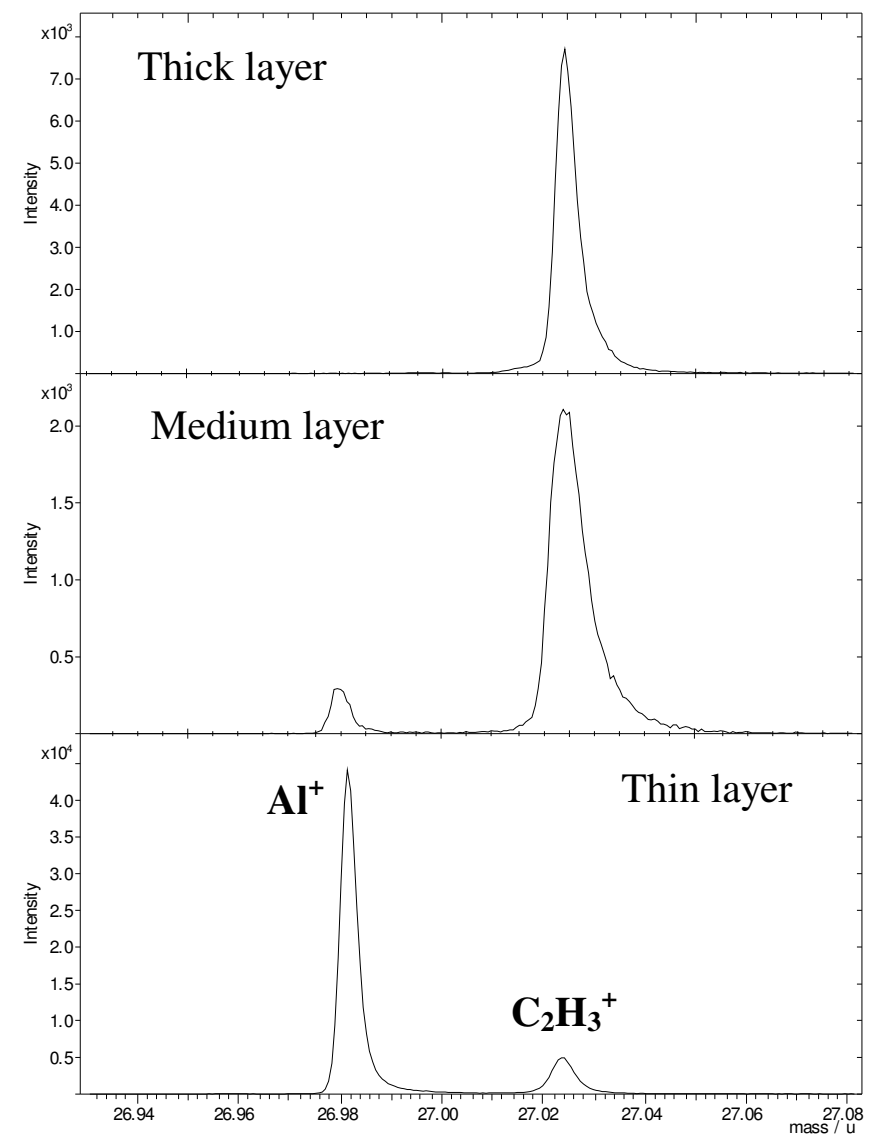

Figure 2: Asymmetry of peaks $\mathrm{C}_{6} \mathrm{H}_{5}{ }^{+}$and $\mathrm{C}_{8} \mathrm{H}_{7}{ }^{+}$as a function of film thickness.

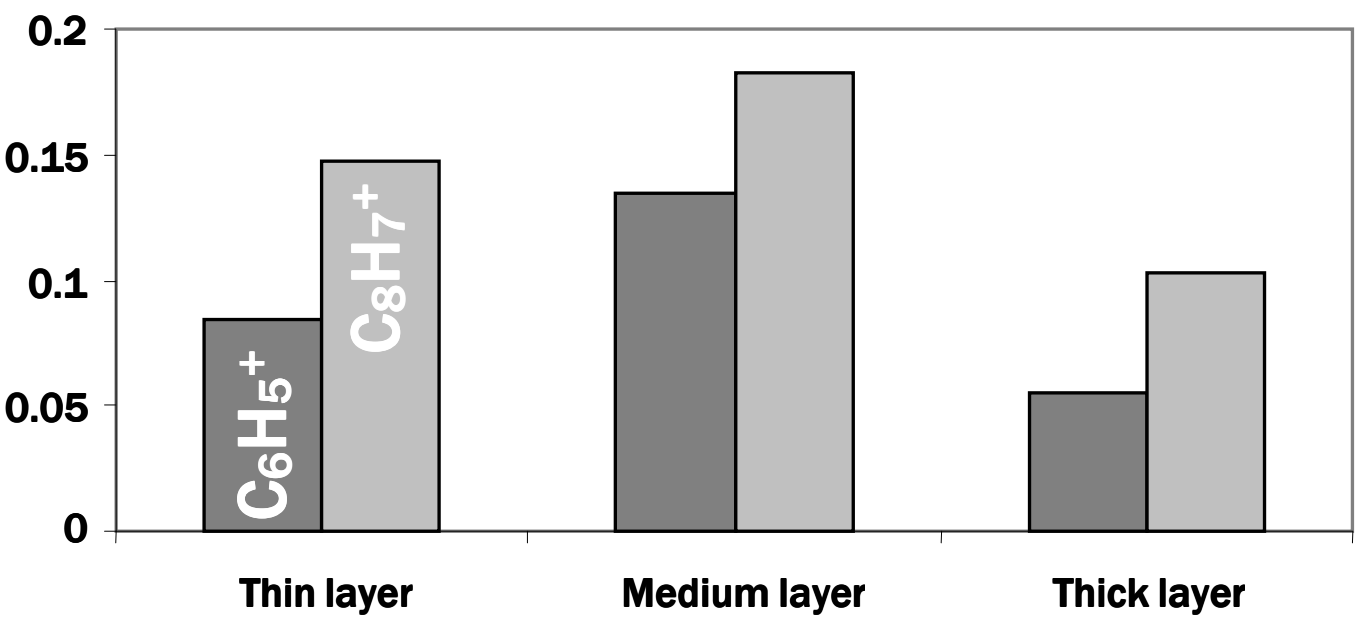


Figure 3: Peak-fitting of nominal mass 71 for an aluminium sample treated with GPS solution $\left(4.5 \times 10^{-1} \mathrm{M}\right)$. The red envelope is the reconstructed signal.

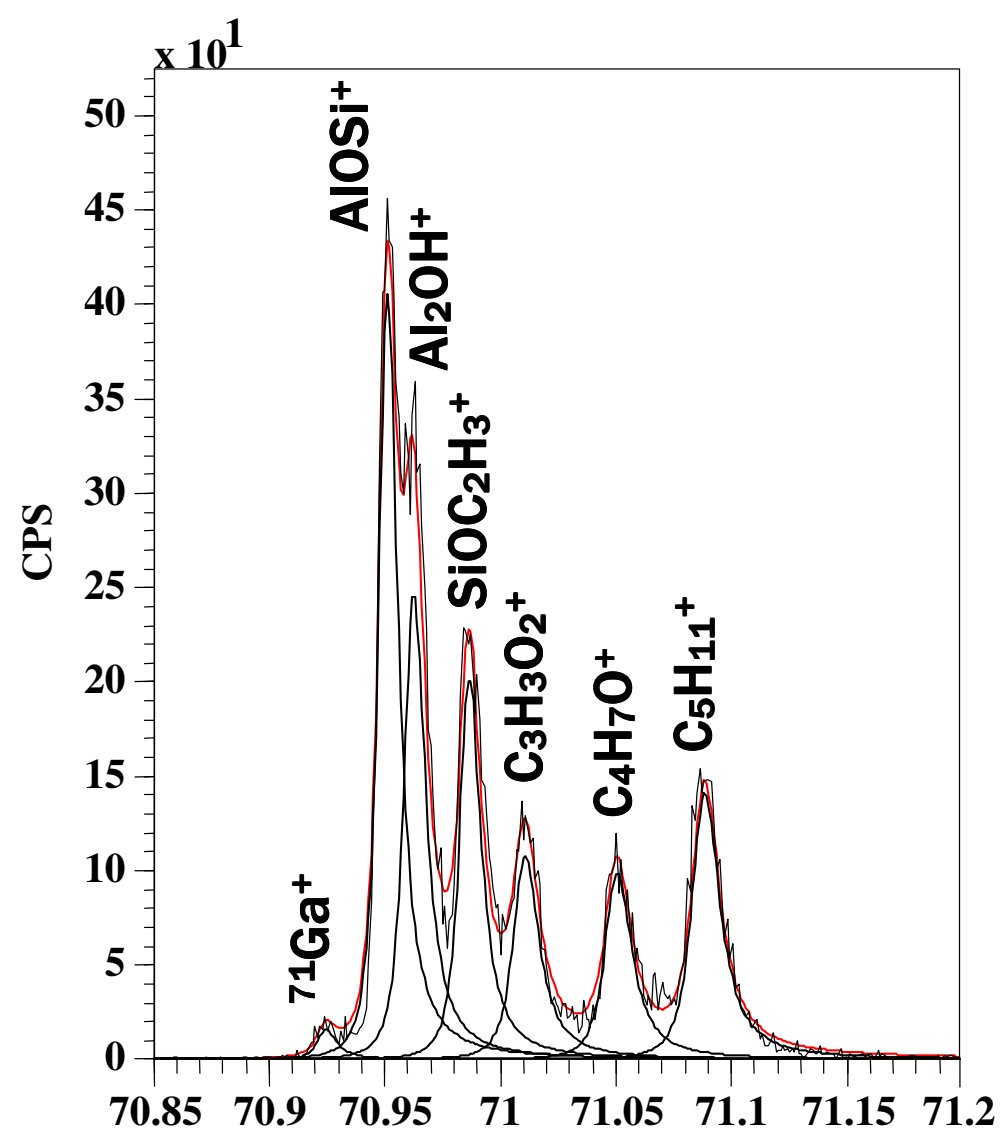


Table 1: Summary of FWHM and peak asymmetry for aluminium samples (signals recorded with a $\mathrm{Bi}_{3}{ }^{+}$primary ion beam)

\begin{tabular}{|c|c|c|c|c|}
\hline \multirow{2}{*}{ Samples } & \multicolumn{2}{|c|}{ FWHM } & \multicolumn{2}{c|}{ Peak asymmetry $(a)$} \\
\cline { 2 - 5 } & $\mathrm{Al}^{+}$ & $\mathrm{Al}(\mathrm{OH})_{2}{ }^{+}$ & $\mathrm{Al}^{+}$ & $\mathrm{Al}(\mathrm{OH})_{2}{ }^{+}$ \\
\hline Polished aluminium & 0.0025 & 0.0069 & -0.119 & -0.118 \\
\hline Aluminium matt side & 0.0023 & 0.0083 & -0.108 & -0.157 \\
\hline Aluminium foil shiny side & 0.0026 & 0.0078 & -0.135 & -0.181 \\
\hline Degreased aluminium & 0.0029 & 0.0079 & -0.153 & -0.271 \\
\hline Grit-blasted aluminium & 0.0037 & 0.0107 & -.0138 & -0.191 \\
\hline Heated aluminium & 0.0035 & 0.0082 & -0.228 & -0.326 \\
\hline
\end{tabular}

Table 2: Summary of FWHM and peak asymmetry for polymer samples (solution concentration $3.4 \times 10^{-4} \mathrm{M}$ for all samples)

\begin{tabular}{|c|c|c|c|c|c|c|}
\hline \multicolumn{7}{|c|}{ Analysis recorded with $\mathrm{Bi}^{+}$primary ion beam } \\
\hline \multirow{2}{*}{ Samples } & $\mathrm{H}^{+}$ & $\mathrm{C}_{6} \mathrm{H}_{5}{ }^{+}$ & $\mathrm{C}_{8} \mathrm{H}_{7}{ }^{+}$ & $\mathrm{H}^{+}$ & $\mathrm{C}_{6} \mathrm{H}_{5}{ }^{+}$ & $\mathrm{C}_{8} \mathrm{H}_{7}{ }^{+}$ \\
\cline { 2 - 7 } & 0.00043 & 0.014 & 0.017 & -0.020 & -0.021 & -0.062 \\
\hline $\mathrm{MW}=90 \mathrm{~K}$ & 0.011 & 0.014 & -0.049 & -0.029 & -0.023 \\
\hline $\mathrm{MW}=233 \mathrm{~K}$ & 0.00042 & 0.012 & 0.015 & -0.082 & -0.134 & -0.176 \\
\hline \multicolumn{7}{|c|}{ Analysis recorded with $\mathrm{Bi}_{3}{ }^{+}$primary ion beam } \\
\hline \multirow{2}{*}{ Samples } & 0.00042 & $0.01 \mathrm{~K}$ & \multicolumn{5}{c|}{ Peak asymmetry $(a)$} \\
\cline { 2 - 7 } & $\mathrm{H}^{+}$ & $\mathrm{C}_{6} \mathrm{H}_{5}{ }^{+}$ & $\mathrm{C}_{8} \mathrm{H}_{7}{ }^{+}$ & $\mathrm{H}^{+}$ & $\mathrm{C}_{6} \mathrm{H}_{5}{ }^{+}$ & $\mathrm{C}_{8} \mathrm{H}_{7}{ }^{+}$ \\
\hline $\mathrm{MW}=90 \mathrm{~K}$ & 0.0052 & 0.013 & 0.013 & -0.084 & -0.024 & -0.078 \\
\hline $\mathrm{MW}=233 \mathrm{~K}$ & 0.0052 & 0.010 & 0.013 & -0.076 & -0.034 & -0.116 \\
\hline $\mathrm{MW}=950 \mathrm{~K}$ & 0.0052 & 0.014 & 0.018 & -0.089 & -0.056 & -0.103 \\
\hline
\end{tabular}

References

[i]. S. R. Leadley, J. F. Watts, J. Adhesion 1997 ; 60175

[ii].S. R. Leadley, J. F. Watts, J. Elec. Spec. 1997 ; 85107

[iii]. K. Shimizu, M-L. Abel, J.F. Watts, unpublished results 2008

[iv]. K. Shimizu, M-L. Abel, J.F. Watts, J. Adhesion 2008 ; 84725

[v]. Ian Gilmore. ToF-SIMS: Surface Analysis by Mass Spectrometry, Eds J.C.

Vickermann and D. Briggs, IMPublications and Surface Spectra, (2001) pp 261-283. 